IDDF2019-ABS-0052 PREVALENCE OF HEPATITIS C AMONG TYPE 2 DIABETES MELLITUS PATIENTS IN A SINGLE CENTER TERTIARY HOSPITAL IN QUEZON CITY, PHILIPPINES

Millette Castro* . University of the East Ramon Magsaysay Memorial Medical Center, Philippines

10.1136/gutjnl-2019-IDDFabstracts.250

Introduction There is a growing body of literature between Hepatitis $C$ virus infection and T2DM on the framework of insulin resistance. There have recent studies showing Diabetes as a strong risk factor for the development of Hepatitis $\mathrm{C}$ in some Asian countries. However, there are still gaps in the literature and data is still inconclusive. In the Philippines, no local study on the prevalence of $\mathrm{HCV}$ among diabetics is available.

This is a pilot study that aims to determine the prevalence of HCV infection among diabetic patients seen at our institution.

Methodology This is a descriptive cross-sectional epidemiological study. Written consent was secured, a consecutive sampling of 318 known diabetics was done and data was collected using a checklist. A drop of whole blood was collected by the investigator by finger prick method and tested for HCV antibodies using RAPIDQuick screening test. Frequency and proportion and median and range were used to describe the clinical characteristics of the enrolled subjects. STATA 15.0 was used for data analysis.

Results 318 diabetic patients were tested for HCV. No HCV seropositivity was noted. The median age was 55 years and a half were female. $22 \%$ were obese. The following risk factors for HCV were reported: more than half had reported unprotected sex, shares nail cutters, toothbrush, and razors. 261 $(82 \%)$ had abnormal FBS and median HbA1c was $7.5 \%$. Similarly, 3/4 had abnormal triglyceride levels. On average, more than half of the patients are insulin-requiring for 4 years have T2DM for four years.

Conclusions Despite a negative study, we would like to continue this study to determine the association between the two diseases and in the future can contribute to the local statistics of Hepatitis C.

\section{IDDF2019-ABS-0063 A DIFFERENCE-IN-DIFFERENCE BASED CT RADIOMICS ALGORITHM FOR DETECTING RECURRENCE OF HEPATOCELLULAR CARCINOMA AFTER RESECTION OR ABLATION: AN EXPLORATORY ANALYSIS}

${ }^{1}$ Zhihang Chen*, ${ }^{2}$ Jingxian Shen, ${ }^{3}$ Qian Zhou, ${ }^{3}$ Sui Peng, ${ }^{1}$ Ming Kuang. ${ }^{1}$ Department of Liver Surgery, The First Affiliated Hospital of Sun Yat-sen University, Guangzhou, China; ${ }^{2}$ Department of Medical Imaging, Sun Yat-sen University Cancer Center, Guangzhou, China; ${ }^{3}$ Clinical Trials Unit, The First Affiliated Hospital of Sun Yat-sen University, Guangzhou, China

\subsection{6/gutjnl-2019-IDDFabstracts.251}

Background Early detection of recurrent hepatocellular carcinoma (HCC) can make early intervention available. This study aimed to find radiomics features to improve the performance of detecting recurrence based on computer tomography (CT). Methods The retrospective study included a training cohort of 35 patients and a validation cohort of 62 patients. A series of follow-up CT images of patients with primary HCC after surgical resection or ablation were collected from January 2009 to April 2018. Tumor and liver regions of interest (ROI) were manually delineated on CT1 (within one month after resection or ablation), CT2 (when suspicious recurrence was observed) and CT3 (when confirmed recurrence was observed). Radiomics features were extracted and a radiomics algorithm, 'difference-in-difference' (DD) was established for detecting recurrence. Folded cross validation (CV) was used. Results A total of 256 follow-up images were available for analysis. The training cohort was divided into recurrence and no recurrence group, with 18 patients and 17 patients, respectively. Our radiomics algorithm selected 17 DD radiomics features for detecting recurrence using univariable analysis. Principle component analysis showed that an Area Under the Receiver Operator Characteristic Curve (AUC) of the first principle component of 17 selected DD features achieved 0.97 (95\% CI: 0.92-1.00). Leave-one-out CV produced an error of $18.2 \%$ and 10 -fold CV with a highest error as $19.1 \%$. Incorporating post-treatment alpha-fetoprotein (AFP), there was no significant increase in AUC compared with the first principal component of $17 \mathrm{DD}$ features alone (0.97 vs $0.97, Z=0.346$, $P=0.730)$. In the validation cohort, univariable analysis showed that the variation trend of radiomics features was suggestive of recurrence.

Conclusions Difference-in-difference of CT radiomics features improved performance of conventional CT and was superior to AFP in detecting recurrence of HCC.

\section{IDDF2019-ABS-0066 NOVEL PROGNOSTIC NOMOGRAMS BASED ON INFLAMMATION-RELATED MARKERS FOR PATIENTS WITH HEPATOCELLULAR CARCINOMA UNDERWENT HEPATECTOMY}

${ }^{1}$ Yifei Wang*, ${ }^{1}$ Kaiyu Sun, ${ }^{2}$ Jingxian Shen, ${ }^{1}$ Bin Li, 'Qinghua Cao, ${ }^{1}$ Sui Peng, ${ }^{1}$ Ming Kuang. ${ }^{1}$ The First Affiliated Hospital of Sun Yat-sen University, China; ${ }^{2}$ Sun Yat-sen University Cancer Center, China

\subsection{6/gutjnl-2019-IDDFabstracts.252}

Background Hepatocellular carcinoma (HCC) is an aggressive disease with high recurrence rate. However, current staging systems were lack of predictive capacity for HCC recurrence after hepatic resection. We aimed to develop prognostic nomograms based on inflammation-related markers for HCC patients underwent hepatectomy.

Methods We retrospectively recruited 1350 surgically treated HCC patients from two medical centres between November 2003 and December 2017. All established inflammation-related markers were enrolled in analysis for selecting the best in predicting recurrence-free survival (RFS) and overall survival (OS). Independent prognostic factors were identified by cox regression analyses. Nomograms for estimating the probability of 1-, 2-, 3-year RFS and OS were established, and validated internally and externally. The performance, discrimination and calibration of nomograms were assessed, and compared with conventional staging systems including Barcelona Clinic Liver Cancer (BCLC) and $7^{\text {th }}$ American Joint Committee on Cancer (AJCC).

Results Gamma-glutamyl transpeptidase to platelet ratio (GPR) was the only inflammation-related factor that independently correlated with both RFS and OS. GPR, international normalized ratio (INR), alpha-fetoprotein (AFP), microvascular invasion, satellite lesions, tumour number, tumour diameter and macrovascular invasion were used to construct nomogram for 